\title{
Circulating miRNA-21 is a promising biomarker for heart failure
}

\author{
JIANGHUA ZHANG ${ }^{*}$, QIANG XING*, XIANHUI ZHOU, JINXIN LI, YAODONG LI, \\ LIN ZHANG, QINA ZHOU and BAOPENG TANG
}

\begin{abstract}
Department of Cardiology, First Affiliated Hospital of Xinjiang Medical University, Ürümqi, Xinjiang 830054, P.R. China
\end{abstract}
Received September 27, 2016; Accepted July 20, 2017

DOI: $10.3892 / \mathrm{mmr} .2017 .7575$

\begin{abstract}
RNA 21 (miRNA-21) promotes the development of cardiac fibrosis, hypertrophy and heart failure. However, whether it can be used as a biomarker for the diagnosis and prognosis of heart failure remains unclear. The current study assessed circulating miRNA-21 as a viable indicator for diagnosis and prognosis of heart failure. The levels of miRNA-21 and brain natriuretic peptide were measured in serum obtained from the peripheral vein (miRNA-21-PV) and coronary sinus (miRNA-21-CS) of 80 patients with heart failure and 40 control individuals via reverse transcription-quantitative polymerase chain reaction and ELISA, respectively. The correlations between circulating miRNA-21 and diagnosis, severity, prognosis and re-hospitalization rate of heart failure were evaluated using statistical analysis. Serum miRNA-21-PV and miRNA-21-CS levels of patients with heart failure were significantly higher than that of control subjects, and were also correlated with ejection fraction and brain natriuretic peptide. Both were determined to have high levels of sensitivity and specificity for diagnosing heart failure. Follow-up of the patients with heart failure indicated that miRNA-21-PV and miRNA-21-CS were correlated with prognosis, and miRNA-21-CS was efficient in predicting re-hospitalization for heart failure. Circulating miRNA-21 has potential to be a biomarker of heart failure.
\end{abstract}

\section{Introduction}

MicroRNAs (miRNAs) are endogenous, non-coding, single-stranded RNAs consisting of fewer than 22 nucleotides, and are encoded by short inverted repeats within the genome $(1,2)$. miRNAs perform their physiological and pathological functions by regulating the expressions of target genes (3). Previous studies have suggested that a number of

Correspondence to: Dr Baopeng Tang, Department of Cardiology, First Affiliated Hospital of Xinjiang Medical University, 137 Liyushan South Road, Ürümqi, Xinjiang 830054, P.R. China E-mail: tangbaopeng@yeah.net

*Contributed equally

Key words: heart failure, microRNA-21, biomarker
miRNAs are involved in the pathogenesis of cardiovascular disease (4-6). One of these is miRNA-21.

miRNA-21 was first identified as a tumor growth enhancer $(7,8)$, however was later observed to be involved in mediating the homeostasis of the cardiovascular system (9-11). Abnormal levels of miRNA-21 contribute to the development of a number of cardiovascular diseases including coronary heart disease, cardiac fibrosis and hypertrophy $(12,13)$. For example, Dong et al (14) identified abundant miRNA-21 in rat hypertrophic cardiac cells, and Thum et al (15) reported that miRNA-21 was significantly upregulated in fibrotic failing hearts, and involved in activating extracellular signal-related kinase-mitogen-activated protein kinase signaling that led to fibroblast proliferation and fibrosis. Conversely, the antagonist of miRNA-21 prevented cardiac hypertrophy and reversed cardiac remodeling (15).

Heart failure (HF) is an end stage of numerous cardiac muscle disorders including cardiac hypertrophy, and is the leading cause of hospitalization in the elderly and death worldwide $(16,17)$. Histologically, the failing heart is accompanied by cardiomyocyte death and fibrosis (18).

miRNA-21 in human HF has been studied previously, in human failing heart tissues, miRNA-21 levels were elevated (19). In a rat HF model, elevated miRNA-21 levels facilitated the development of HF, at least in part by promoting cardiac fibrosis (20). Thus, miRNA-21 in the heart contributes to the pathogenesis of HF. However, while miRNA-21 is produced predominantly in the heart, it is unclear whether circulating miRNA-21 can serve as an indicator for HF in human patients.

Identification of specific, reliable and sensitive biomarkers for human diseases is a field of active research. In the cardiovascular field, several molecules have been identified as putative biomarkers for different cardiovascular diseases or different stages of a particular cardiovascular disease. For example, it is well documented that high levels of low-density lipoprotein cholesterol are closely associated with increased risk of coronary heart disease (21). Circulating brain natriuretic peptide (BNP), which is predominantly produced in heart ventricles in response to stress, serves as a sensitive predictor for cardiac dysfunction and for vascular patients with major adverse cardiac events $(22,23)$.

The identification of circulating miRNAs as specific biomarkers for cardiovascular disorders has been researched previously. For example, circulating miRNA-208 and miRNA-150 have been identified as potentially promising 
biomarkers for both agonist-induced cardiac hypertrophy and cardiac remodeling following acute myocardial infarction $(24,25)$. A number of miRNAs, including miRNA-21, have been observed to be elevated in the serum of elderly patients with non-ST segment myocardial infarction (26), suggesting that miRNA-21 may serve as a biomarker for coronary heart diseases.

Given the pathogenic role that miRNA-21 has in the development of $\mathrm{HF}$, and that increased levels of miRNA-21 have been demonstrated in failing hearts in humans and animal models, the present study explored an association between the levels of circulating miRNA-21 and human HF. The observations of the current study provided evidence that miRNA-21 could be a promising biomarker for diagnosis and prognosis of human HF.

\section{Materials and methods}

Study subjects. The Institutional Review Board of First Affiliated Hospital of Xinjiang Medical University approved the study protocol. A total of 120 patients among those hospitalized between March 2013 and October 2013 met the inclusion criteria of the study, signed an informed consent form, and were subsequently recruited into the study. All patients underwent coronary angiogram, radiofrequency ablation or cardiac resynchronization therapy (CRT). Due to the fact that previous studies indicated that serum miRNA-21 levels were high in patients with malignant tumors $(7,27,28)$, the current study excluded patients with malignant tumors.

Among the patients recruited, 40 had normal cardiac function and were placed in the control group, and 80 had abnormal cardiac function and were placed in the HF group. The diagnosis of HF met the guidelines of the 2012 European Society of Cardiology for heat failure (29). The patients in the control group had arrhythmia or angina. The inclusion criteria for HF were: Cardiomyopathy; HF as the first-listed diagnosis; a left ventricular ejection fraction $(\mathrm{LVEF})<50 \%$; and a history of HF for $>6$ months. The exclusion criterion for the HF group was the presence of a malignant tumor. The inclusion criteria of the control group were: $\mathrm{LVEF} \geq 50 \%$; and no symptoms of HF. The exclusion criteria for the control group were: A history of myocardial infarction; or presence of a malignant tumor.

Blood sample collection. All subjects underwent either coronary angiography or radiofrequency catheter ablation. Sheathes were placed in the femoral vein and blood samples were extracted from the coronary sinus (CS) and femoral vein using a coronary angiographic catheter. The patients in the HF group were further stratified into 4 subgroups based on the New York Heart Association (NYHA) Functional Classification. All patients with HF were treated with anti-HF drugs, and their cardiac functions were improved to NYHA II. When the blood was being collected from the CS the X-ray position was at 30 degrees, relative to the left anterior oblique position.

Under fluoroscopy, the catheter reached the upper right atrium and turned toward the spine. The operator then slowly pulled back the catheter, jumping of the catheter signified that the catheter's tip had reached the CS. The operator used a contrast agent during angiography to ensure that the catheter was localized in the coronary sinus.
Serum isolation and storage. A total of 2 6-8 $\mathrm{ml}$ blood samples were separately collected from the CS and femoral vein of each subject. The collection tube (BD 762165 PAXgene Blood RNA tube) was purchased from BD Biosciences (Franklin Lakes, NJ, USA). All blood samples were allowed to stand for less than $2 \mathrm{~h}$ prior to being centrifuged at $1,800 \mathrm{x}$ for $10 \mathrm{~min}$, after which the serum samples were collected and stored at $-80^{\circ} \mathrm{C}$.

Measurement of BNP. The level of BNP was measured using a human BNP enzyme-linked immunosorbent assay kit (cat. no. AK0014JUN09001; Elabscience Biotechnology Co., Ltd., China). The measurement of each sample was conducted in triplicate. Testers of the samples were blinded to the study group.

RNA extraction and cDNA synthesis. Total RNA was extracted from serum using the TRIzol LS reagent (Invitrogen; Thermo Fisher Scientific, Inc., Waltham, MA, USA) based on the protocol provided by the manufacturer. Briefly, 0.25-ml serum samples were homogenized in $0.75 \mathrm{ml}$ TRIzol reagent and $0.2 \mathrm{ml}$ chloroform was added to each sample. All samples were centrifuged at $12,000 \times \mathrm{g}$ for $15 \mathrm{~min}$ at $4^{\circ} \mathrm{C}$ to separate the mixture. RNA in the aqueous phase was precipitated with $0.5 \mathrm{ml}$ isopropyl alcohol. Following centrifugation, the pellets (i.e., RNA) were washed with $75 \%$ ethanol. RNA purity was determined from the $260 / 280 \mathrm{~nm}$ ratio, and only those samples with a ratio of between 1.8 and 2.1 were used in the present study.

Reverse transcription (RT) was performed using $4 \mu \mathrm{g}$ of total RNA in a total reaction volume of $20 \mu \mathrm{l}$, with the RevertAid First Strand cDNA Synthesis kit (Fermentas; Thermo Fisher Scientific, Inc.) using hsa-mir-21 RT primer 5'-GTCGTATCCAGTGCAGGGTCCGAGGTA TTCGCACTGGATACGACTCAACATC-3'. Briefly, subsequent to an initial treatment at $70^{\circ} \mathrm{C}$ for $5 \mathrm{~min}$, the RT reaction was conducted at $42^{\circ} \mathrm{C}$ for $1 \mathrm{~h}$ using $1 \mu \mathrm{l}$ Moloney murine leukemia virus reverse transcriptase, and terminated by heating to $92^{\circ} \mathrm{C}$ for $5 \mathrm{~min}$. Following cDNA synthesis, all cDNA samples were diluted 10 times in molecular grade water and stored at $-20^{\circ} \mathrm{C}$.

Quantitative polymerase chain reaction ( $q P C R)$. qPCR was performed using an ABI viia7 Sequence Detection system (Applied Biosystems; Thermo Fisher Scientific, Inc.). The hsa-mir-21 PCR primers were: Forward, 5'-TGCGCTAGC TTATCAGACTGA-3' and reverse, 5'-CCAGTGCAGGGT CCGAGGTATT-3'. U6 small nuclear RNA was used as an endogenous control for normalization. The qPCR reaction contained $10 \mu \mathrm{l}$ 2X SYBR-Green/Fluorescein qPCR Master mix (Fermentas; Thermo Fisher Scientific, Inc.), the forward and reverse primers, RNase-free water and $2 \mu \mathrm{l}$ cDNA template per reaction in a final volume of $20 \mu \mathrm{l}$. The thermocycling conditions for quantitative PCR were 1 cycle of $50^{\circ} \mathrm{C}$ for $2 \mathrm{~min}, 95^{\circ} \mathrm{C}$ for $10 \mathrm{~min}, 40$ cycles of $30 \mathrm{sec}$ at $94^{\circ} \mathrm{C}$ and $30 \mathrm{sec}$ at $60^{\circ} \mathrm{C}$.

The data were processed using the relative quantification method. The relative values were measured as $2^{-\Delta \Delta \mathrm{Ct}}(30)$. Testers were blinded to the patient group. The expression levels relative to U6 were $18.88 \pm 1.49$ in the vein and $28.10 \pm 1.49$ in 
the coronary of the control group, and $20.01 \pm 0.82$ in the vein and $28.94 \pm 1.07$ in the coronary of the HF group.

Data analysis and statistics. Data were analyzed using the statistical software package SPSS 17.0 (SPSS, Inc., Chicago, IL, USA). Continuous clinical variables were compared between the 2 groups using a two-sided unpaired t-test. Analysis of covariance was used to analysis the effect of confounding factors. The Chi-square test was applied to compare categorical clinical variables. Receiver operating characteristic (ROC) curve analysis was used to evaluate the association between miRNA-21 levels and a diagnosis of HF. Pearson's correlation coefficient was employed to evaluate the correlation between clinical features and serum miR-21. Binary logistic regression was used to evaluate the correlation between serum miRNA-21 and other factors with the re-hospitalization rate for HF. A COX regression model was used to evaluate the correlation between serum miRNA-21 and other factors with the prognosis of HF. Continuous clinical variables were presented as the mean \pm standard deviation. $\mathrm{P}<0.05$ was considered to indicate a statistically significant difference.

\section{Results}

Demographic and clinical characteristics of the $H F$ and control groups. The HF and control groups were significantly different with regard to age, gender, pathogenesis, hemoglobin, systolic pressure, renal function and levels of BNP and miRNA-21 in the peripheral vein (PV) and CS (Table I).

Increased circulating BNP and miRNA-21 in HF patients. A number of studies have indicated a higher level of BNP in the CS than in other tissues of the body $(31,32)$, and it has been well documented that the majority of circulating BNP originates from the heart (33). Consistent with the above observations, in the present study BNP levels in the CS were higher than in the PV in patients of either the HF or control group (Fig. 1A). Notably, the data of the present study indicated that BNP levels from either the PV or CS in HF patients were significantly higher than that of the control group.

The levels of circulating miRNA-21 in the PV were lower in the CS in both the HF and control groups, and circulating miRNA-21 in the patients with HF was significantly higher, than that of the control group (Fig. 1B). Previous studies have indicated that age (34) and diabetes (35) affected the expression of miRNA-21. Therefore, analysis of covariance was used to analyze the effect of age and diabetes. It was identified that in the samples investigated, age and diabetes did not significantly correlate with the circulating levels of miRNA-21, in either the vein or coronary (Table II).

Levels of miRNA-21 in ischemic cardiomyopathy and dilated cardiomyopathy. Ischemic cardiomyopathy and dilated cardiomyopathy are two main causes of HF. In the HF group, 41 patients had ischemic cardiomyopathy and 33 had dilated cardiomyopathy. There was no significant difference in the EF between the ischemic cardiomyopathy and dilated cardiomyopathy groups $(37.90 \pm 4.01$ vs. $36.39 \pm 4.86 \% ; \mathrm{P}=0.148)$. The levels of miRNA-21-PV and miRNA-21-CS in the two groups were not significantly different (miRNA-21-PV, 3.95 \pm 2.84 vs. $4.13 \pm 2.42, \mathrm{P}=0.773$; miRNA-21-CS, $18.48 \pm 13.28$ vs. 15.46 $\pm 10.81, \mathrm{P}=0.296$; Fig. 2).

Correlation between miRNA-21 and other clinical indices. Correlation analyses were conducted to evaluate associations between serum miRNA-21 and EF, NYHA classification or BNP in the HF group relative to the control group (Fig. 3). miRNA-21-PV was negatively correlated with $\mathrm{EF}(\mathrm{r}=-0.540$, $\mathrm{P}<0.001)$, however positively correlated with NYHA grade $(\mathrm{r}=0.580, \mathrm{P}<0.001)$ and BNP-PV $(\mathrm{r}=0.760, \mathrm{P}<0.001)$. The associations between miRNA-21-CS and $\mathrm{EF}(\mathrm{r}=-0.556, \mathrm{P}<0.001)$, NYHA classification $(\mathrm{r}=0.755, \mathrm{P}<0.001)$ and BNP-CS $(\mathrm{r}=0.725$, $\mathrm{P}<0.001$ ) followed the same pattern as that of miRNA-21-PV to these variables (Fig. 4).

Correlation between miRNA-21 and the severity of $H F$ symptoms. The levels of circulating miRNA-21-PV and miRNA-21-CS gradually increased with increasing NYHA grade (Fig. 5). Analysis of variance indicated that the levels of circulating miRNA-21-PV and miRNA-21-CS were significantly different in each group, excluding that between the NYHA I and II groups.

Correlation between circulating miRNA-21 and diagnosis of HF. The correlation between circulating miRNA-21 and HF was identified by the ROC curve (Fig. 6). Using a threshold score of 1.98 , patients with a score $>1.98$ were predicted to have HF. Using miRNA-21-PV, a sensitivity of $100 \%$, a specificity of $97.5 \%$ and area under curve (AUC) $=0.948$ was achieved for the identification of patients with HF. Similar results were obtained for miRNA-21-CS (sensitivity $=100 \%$, specificity $=97.5 \%$ and $\mathrm{AUC}=0.940$ ).

Correlation between circulating miRNA-21 and prognosis of $H F$. The association between circulating miRNA-21 and the prognosis of the HF group was analyzed using a Cox regression model. The patients with HF were followed for 2 years (mean, $18.33 \pm 3.882$ months). The endpoint of follow-up was death. A total of 17 patients of the HF group died, 1 as a result of a traffic accident, 1 from hypoxemia of pulmonary fibrosis and infection, 12 from cardiac sudden death, and 3 from multiple organ failure caused by HF. EF, BNP-PV, creatinine, CRT and miRNA-21-PV/miRNA-21-CS were included in the Cox regression model. miRNA-21-PV significantly correlated with the endpoint [relative risk $(\mathrm{RR})=1.936,95 \%$ confidence interval $(\mathrm{CI})=1.310-2.865, \mathrm{P}=0.001]$, as did miRNA-21-CS $(\mathrm{RR}=1.125$, 95\% CI=1.049-1.1206, $\mathrm{P}=0.001$; Table III).

Correlation between circulating miRNA-21-CS and re-hospitalization rate for $H F$. A binary logistic regression model was used to evaluate a correlation between serum miRNA- 21 and other factors and the re-hospitalization rate for HF. A total of 23 of the 60 patients with HF required re-hospitalization during the follow-up period.

EF, BNP-PV, creatinine, CK-MB, alanine aminotransferase, CRT, miRNA-21-PV and miRNA-21-CS were analyzed using binary logistic regression. Similar to the analysis with the Cox regression model, the miRNA-21-PV and miRNA-21-CS serum levels were analyzed to compare their correlations with re-hospitalization rates for HF. miRNA-21-PV exhibited no significant 
Table I. Demographics and clinical characteristics of the HF and control groups.

\begin{tabular}{|c|c|c|c|}
\hline Category & $\mathrm{HF}$ & Control & P-value \\
\hline Subjects & 80 & 40 & \\
\hline Gender, male/female & $57 / 23$ & $20 / 20$ & 0.027 \\
\hline Age, years & $59.68 \pm 10.24$ & $53.45 \pm 12.40$ & 0.04 \\
\hline Weight, kg & $69.50 \pm 12.80$ & $70.42 \pm 16.08$ & 0.752 \\
\hline Dilated cardiomyopathy & 33 & 0 & \\
\hline Ischemic cardiomyopathy & 41 & 0 & \\
\hline Hypertensive cardiomyopathy & 4 & 0 & \\
\hline Valvular heart disease & 2 & 0 & \\
\hline PSVT & 0 & 21 & \\
\hline Premature RVOT & 0 & 3 & \\
\hline Coronary heart disease & 41 & 10 & 0.007 \\
\hline Physical examination & 0 & 5 & \\
\hline Hypertension & 23 & 12 & 0.877 \\
\hline Diabetes mellitus & 20 & 5 & 0.001 \\
\hline Atrial fibrillation & 9 & 1 & 0.089 \\
\hline History of heart failure, years & $2.72 \pm 2.78$ & - & \\
\hline NYHA I & 11 & - & \\
\hline NYHA II & 22 & - & \\
\hline NYHA III & 39 & - & \\
\hline NYHA IV & 8 & - & \\
\hline ACEI/ARB & 80 & 0 & \\
\hline Beta blocker & 77 & 1 & \\
\hline Aldospirone & 55 & 0 & \\
\hline Amiodarone & 9 & 1 & 0.139 \\
\hline CRT & 20 & 0 & \\
\hline Systolic blood pressure, $\mathrm{mmHg}$ & $115.54 \pm 18.40$ & $125.98 \pm 14.40$ & 0.02 \\
\hline Diastolic blood pressure, $\mathrm{mmHg}$ & $71.13 \pm 12.62$ & $75.70 \pm 10.70$ & 0.052 \\
\hline Hemoglobin, g/l & $132.53 \pm 15.21$ & $137.20 \pm 15.39$ & 0.117 \\
\hline Creatinine, mmol/1 & $79.31 \pm 25.93$ & $61.55 \pm 15.30$ & 0 \\
\hline Alanine aminotransferase, $\mathrm{U}$ & $31.16 \pm 18.86$ & $28.27 \pm 4.14$ & 0.439 \\
\hline $\mathrm{CK}-\mathrm{MB}, \mathrm{U} / 1$ & $14.41 \pm 7.41$ & $13.09 \pm 4.83$ & 0.308 \\
\hline LVED, mm & $63.07 \pm 7.79$ & $47.75 \pm 3.13$ & 0 \\
\hline Left atrial diameter, mm & $42.18 \pm 4.98$ & $33.85 \pm 4.14$ & 0 \\
\hline LVEF, $\%$ & $37.76 \pm 4.98$ & $61.85 \pm 5.43$ & 0 \\
\hline BNP-PV, pg/ml & $767.34 \pm 362.75$ & $199.80 \pm 75.43$ & 0 \\
\hline BNP-CS, pg/ml & $1,277.43 \pm 515.61$ & $319.82 \pm 122.77$ & 0 \\
\hline miRNA-21-PV & $3.98 \pm 2.59$ & $0.90 \pm 0.62$ & 0 \\
\hline miRNA-21-CS & $17.25 \pm 12.28$ & $3.81 \pm 3.24$ & 0 \\
\hline
\end{tabular}

Values are reported as n, unless otherwise indicated. HF, heart failure; PSVT, paroxysmal superaventricular tachycardia; RVOT, right ventricular outflow tract ventricular; NYHA, New York Heart Association; ACEI, angiotensin converting enzyme inhibitor; ARB, angiotensin receptor blocker; CRT, cardiac resynchronization therapy; CK-MB, creatine kinase, muscle and brain; LVED, left ventricular end diastolic diameter; $\mathrm{LVEF}$, left ventricular ejection fraction; BNP, brain natriuretic peptide; PV, peripheral vein; CS, coronary sinus.

association with re-hospitalization rate [overall risk $(\mathrm{OR})=1.001$, 95\% CI=0.993-1.010, $\mathrm{P}=0.757]$, however miRNA-21-CS did $(\mathrm{OR}=1.160,95 \% \mathrm{CI}=1.023-1.315, \mathrm{P}=0.021$; Table IV $)$.

\section{Discussion}

In the present study, it was investigated whether miRNA-21 could serve as a valuable predicator for HF. The current study established, to the best of our knowledge for the first time, that circulating miRNA-21 may not only be a promising biomarker of human HF, however additionally an efficient predictor of mortality and re-hospitalization of patients with HF.

Similar to serum BNP, serum miRNA-21 levels taken from the CS were significantly higher than that from the PV, regardless of whether the samples were from the control or HF patients. This suggests that the heart is an important source 
Table II. Age and diabetes were not correlated with circulating microRNA-21 levels in patients with heart failure as analyzed by analysis covariance.

\begin{tabular}{|c|c|c|c|c|}
\hline \multirow[b]{2}{*}{ Category } & \multicolumn{2}{|c|}{ Vein } & \multicolumn{2}{|c|}{ Coronary } \\
\hline & P-value & $95 \%$ CI & P-value & $95 \%$ CI \\
\hline Heart failure & $<0.001$ & $2.197-3.926$ & $<0.001$ & $8.669-16.815$ \\
\hline Age & 0.796 & $-0.32-0.42$ & 0.484 & $-0.112-0.236$ \\
\hline Diabetes & 0.993 & $-1.007-0.997$ & 0.219 & $-2.238-7.029$ \\
\hline
\end{tabular}

CI, confidence interval.

A

From peripheral vein
From coronary sinus

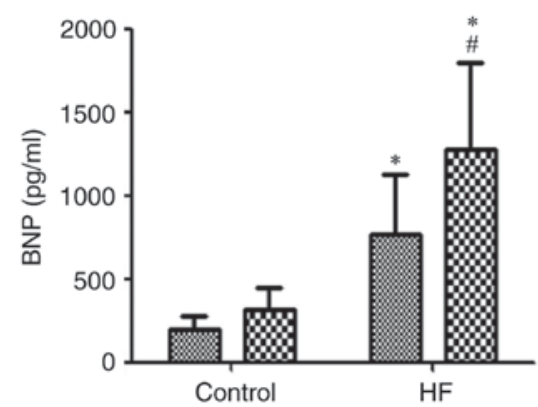

B
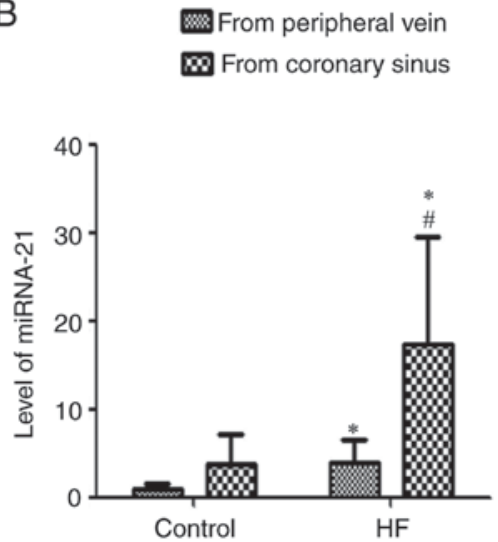

Figure 1. Increased levels of BNP and miRNA-21 in the PV and coronary sinus of patients with HF. (A) BNP; (B) miRNA-21. "P<0.05 vs. the respective control group; ${ }^{~} \mathrm{P}<0.05$ vs. the $\mathrm{PV}$ of the HF group. BNP, brain natriuretic peptide; miRNA, microRNA; HF, heart failure; $\mathrm{PV}$, peripheral vein.

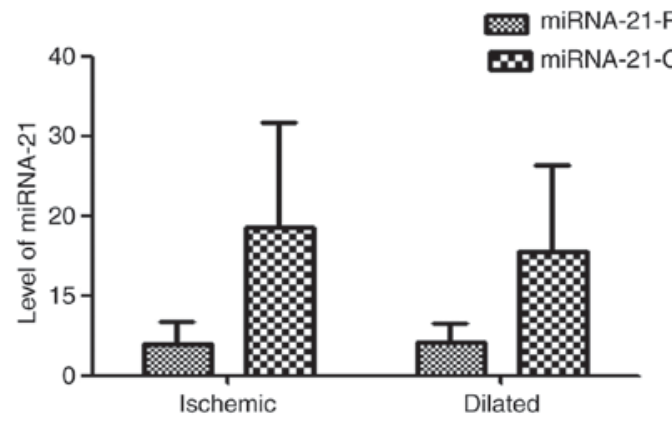

Figure 2. No significant difference was observed in the levels of miRNA-21-PV and miRNA-21-CS between ischemic cardiomyopathy and dilated cardiomyopathy groups. $\mathrm{P}>0.05$. miRNA, microRNA; PV, peripheral vein; $\mathrm{CS}$, coronary sinus.

of serum miRNA-21 in patients with HF. By contrast, it was identified that the levels of circulating miRNA-21 in the PVs and CSs of the HF group were significantly higher than that of the control group, indicating that serum miRNA-21 may be a predictor of HF. Notably, a blood sample obtained from one patient in the control group had a significantly higher level of circulating miRNA-21 relative to the other control samples, and it was determined that this control subject had atrial fibrillation. Thus, consistent with a previous report, miRNA-21 may be involved in the pathogenesis of atrial fibrillation (36).

For a factor to qualify as a biomarker of HF, the expression of this factor should not be significantly altered in the failing hearts caused by different forms of cardiac diseases. Ischemic cardiomyopathy and dilated cardiomyopathy are the two most common causes of HF. In the present study, there was no significant difference in EF between these two groups. Further analysis identified that the circulating levels of miRNA-21-CS and miRNA-21-PV additionally exhibited no significant difference between these two groups, suggesting that the circulating miRNA-21 was not significantly altered due to different causes of HF, i.e., ischemic or non-ischemic cardiomyopathy.

Due to the fact that significantly higher circulating levels of miRNA-21-CS and miRNA-21-PV were identified in patients with HF compared with the control subjects, associations between serum miRNA-21 and cardiac function status, diagnosis, prognosis or re-hospitalization rates were investigated. It was observed that circulating miRNA-21-PV and miRNA-21-CS correlated well and linearly with cardiac EF, BNP and NYHA grade; the latter evidenced by a gradual increase in levels with increasing NYHA grade. However, the association between miRNA-21-CS and EF was closer than that of the association between miRNA-21-PV and EF, indicating that miRNA-21-CS may be a more effective indicator of cardiac function and HF. miRNA-21-PV and miRNA-21-CS exhibited similar efficacy in the diagnosis of HF, as similar sensitivity (100\%) and specificity (97.5\%) were observed, as indicated by the ROC curve.

Few clinical studies have investigated the potential of miRNAs as an indicator of prognosis in HF. In the present study, the results indicated that serum miRNA-21 was useful 

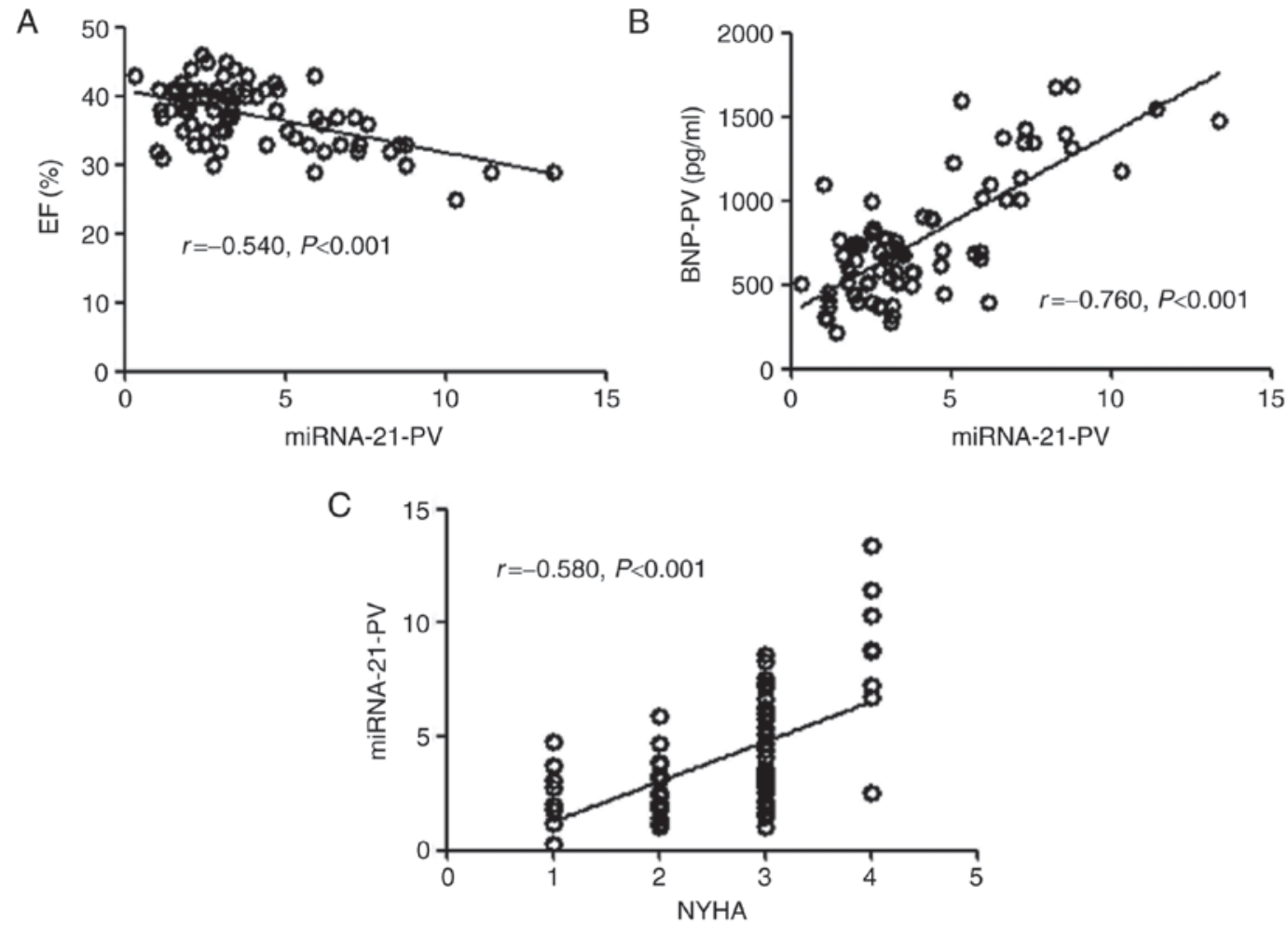

Figure 3. Correlation of miRNA-21-PV with (A) EF, (B) BNP-PV and (C) NYHA grade. miRNA, microRNA; PV, peripheral vein; EF, ejection fraction; BNP, brain natriuretic peptide; NYHA, New York Heart Association.
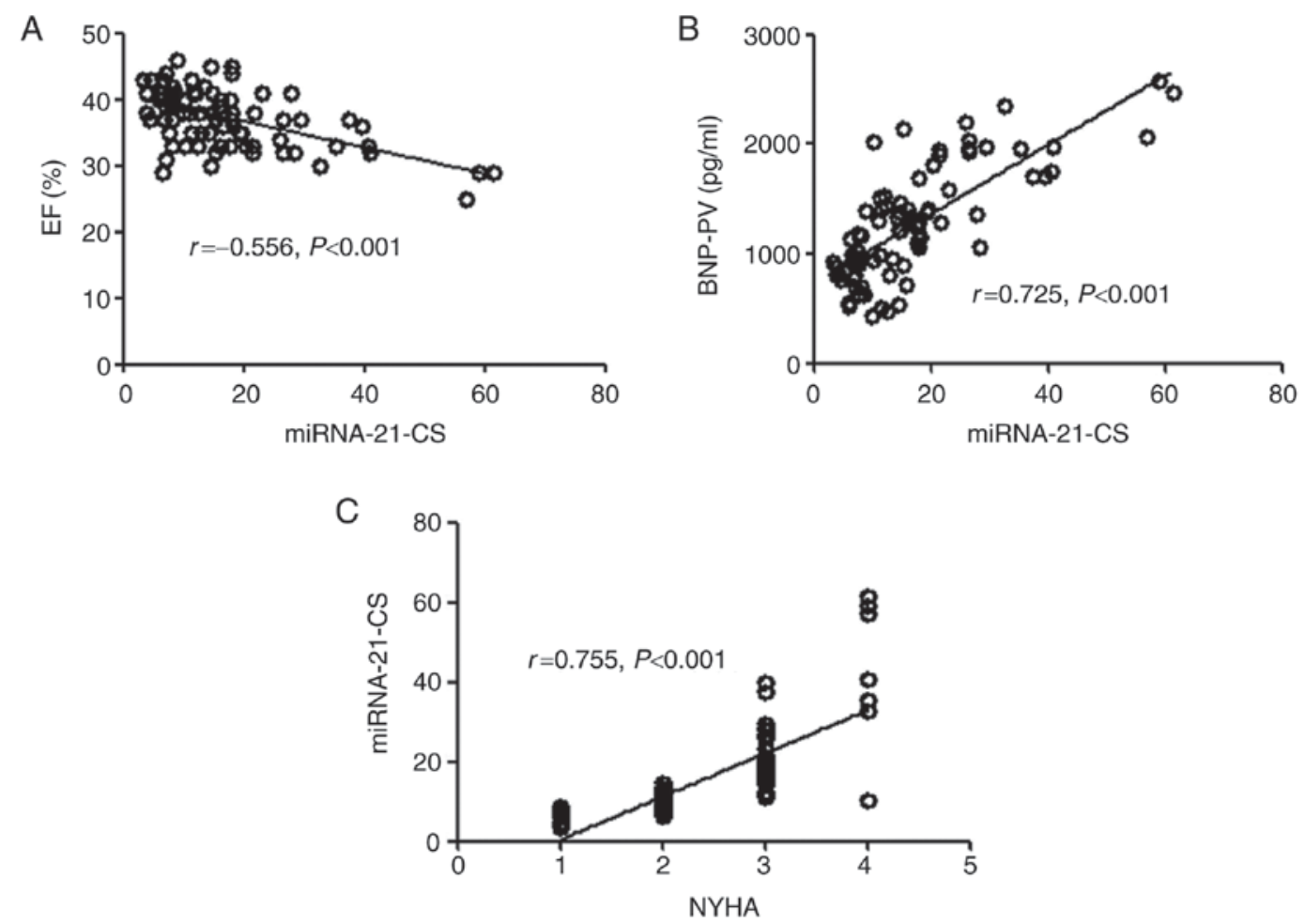

Figure 4. Correlation of miRNA-21-CS with (A) EF, (B) BNP-PV and (C) NYHA grade. miRNA, microRNA; CS, coronary sinus; EF, ejection fraction; BNP, brain natriuretic peptide; PV, peripheral vein; NYHA, New York Heart Association.

for predicting mortality associated with HF. miRNA-21-PV and miRNA-21-CS were significantly associated with mortality of HF patients. However, miRNA-21-CS, but not miRNA-21-PV, was closely associated with re-hospitalization after normalization of EF, BNP, creatinine, CK-MB, CRT and alanine aminotransferase.

Previous studies have demonstrated that CRT improved the quality of life and prognosis of patients with HF (37). 
Table III. Circulating miRNA-21 levels in peripheral veins and the coronary sinus were significantly correlated with the endpoint as analyzed by the Cox regression model.

\begin{tabular}{|c|c|c|c|c|c|c|}
\hline \multirow[b]{2}{*}{ Category } & \multicolumn{3}{|c|}{ Vein } & \multicolumn{3}{|c|}{ Coronary } \\
\hline & P-value & RR & $95 \%$ CI & P-value & RR & $95 \%$ CI \\
\hline $\mathrm{EF}$ & 0.305 & 0.875 & $0.677-1.120$ & 0.196 & 0.850 & $0.665-1.088$ \\
\hline BNP & 0.103 & 1.002 & $1.000-1.005$ & 0.007 & 1.003 & $1.001-1.005$ \\
\hline CRT & 0.218 & 0.348 & $0.065-1.807$ & 0.237 & 0.384 & $0.078-1.875$ \\
\hline miRNA-21 & 0.001 & 1.936 & $1.310-2.862$ & 0.001 & 1.125 & $1.049-1.206$ \\
\hline
\end{tabular}

miRNA-21, microRNA-21; RR, relative risk; CI, confidence interval; LVEF, left ventricular ejection fraction; BNP, brain natriuretic peptide; CRT, cardiac resynchronization therapy.

Table IV. Circulating miR-21 levels in coronary sinus however not in peripheral veins correlated with re-hospitalization rate.

\begin{tabular}{|c|c|c|c|c|c|c|}
\hline \multirow[b]{2}{*}{ Category } & \multicolumn{3}{|c|}{ Vein } & \multicolumn{3}{|c|}{ Coronary } \\
\hline & $\mathrm{P}$-value & OR & $95 \% \mathrm{CI}$ & $\mathrm{P}$-value & OR & $95 \% \mathrm{CI}$ \\
\hline Creatinine & 0.497 & 0.992 & $0.969-1.016$ & 0.508 & 0.991 & $0.965-1.018$ \\
\hline ALT & 0.868 & 0.997 & $0.996-1.030$ & 0.902 & 1.002 & $0.968-1.038$ \\
\hline CK-MB & 0.315 & 1.084 & $0.926-1.270$ & 0.575 & 1.048 & $0.890-1.235$ \\
\hline $\mathrm{EF}$ & 0.073 & 0.896 & $0.745-1.013$ & 0.401 & 0.930 & $0.786-1.101$ \\
\hline BNP & 0.007 & 1.003 & $1.001-1.005$ & 0.384 & 1.001 & $0.999-1.004$ \\
\hline CRT & 0.511 & 0.598 & $0.129-2.766$ & 0.480 & 1.947 & $0.306-12.379$ \\
\hline miR-21 & 0.757 & 1.001 & $0.993-1.010$ & 0.021 & 1.160 & $1.023-1.315$ \\
\hline
\end{tabular}

miR, microRNA; OR, overall risk; CI, confidence interval; ALT, alanine aminotransferase; CK-MB, CK-MB, creatine kinase; LVEF, left ventricular ejection fraction; BNP, brain natriuretic peptide; CRT, cardiac resynchronization therapy.

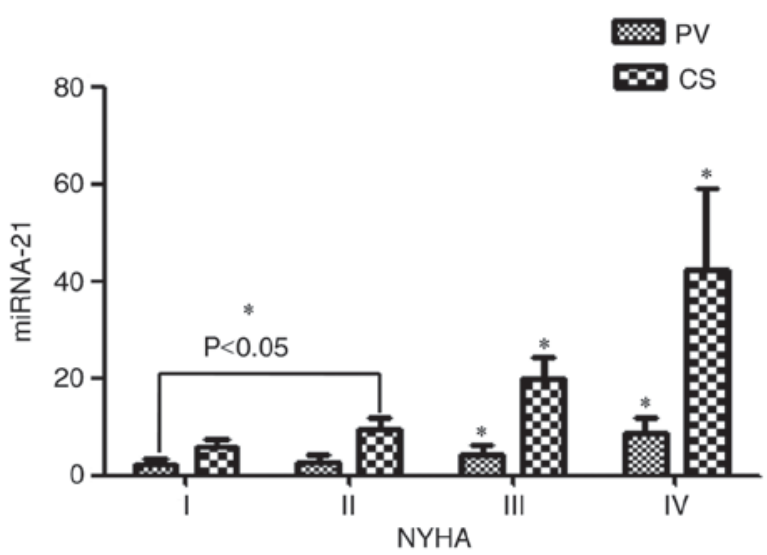

Figure 5. Association of circulating miRNA-21-PV and miRNA-21-CS with NYHA grade. ${ }^{*} \mathrm{P}<0.05$. miRNA, microRNA; PV, peripheral vein; CS, coronary sinus; NYHA, New York Heart Association.

However, in the present study, CRT did not significantly affect prognosis or re-hospitalization. The discrepancy between previous results and those of the current study were likely due to the HF group in the present study including patients with various stages of HF, of which only $25 \%$ of those with severe HF received CRT. Since the heart is the major source of circulating miRNA-21 in patients with HF, it is understandable

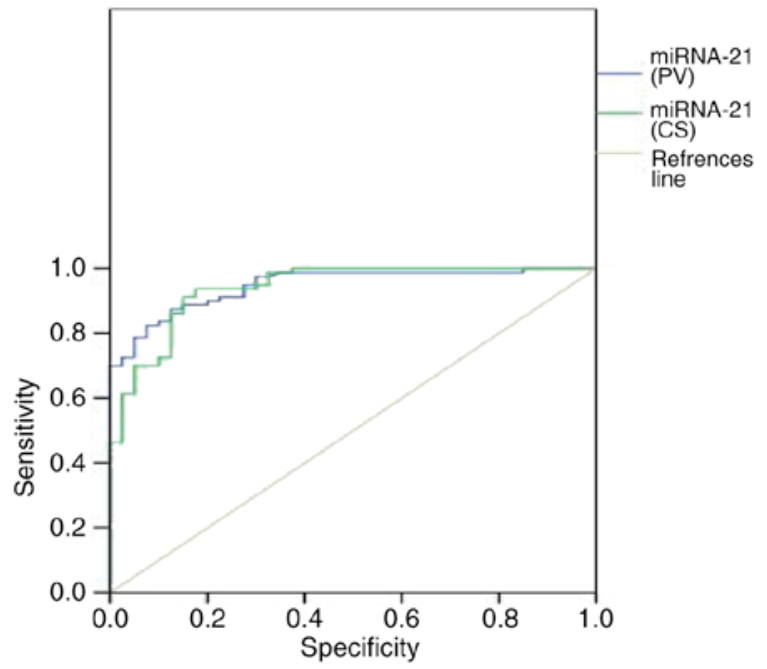

Figure 6. Receiver operating characteristic curve of miRNA-21-PV and miRNA-21-CS for diagnosis of heart failure. miRNA, microRNA; PV, peripheral vein; $\mathrm{CS}$, coronary sinus.

that the level of miRNA-21 in the CS is higher than in the PV, and therefore is probably a more sensitive predicator. However, it was suggested that circulating miRNA-21-PV was sufficient for predicting cardiac function, diagnosis and prognosis of HF. 
Given that circulating miRNA-21-PV is more readily available than miRNA-21-CS, it is anticipated that miRNA-21-PV will have a more practical application in the clinical setting.

The limitations of the present study were as follows. The control group did not match the HF group in terms of age and gender, in addition, the subjects selected for the control group were not healthy people, which may have led to unpredictable effects on the results. In addition, the size of the samples used in the study was small. Thus, further studies with a larger sample size and an improved control group are required in order to corroborate the results.

In summary, the present study compared the levels of circulating miRNA-21 in the PVs and CS of patients with $\mathrm{HF}$ and control subjects. It was determined that both miRNA-21-PV and miRNA-21-CS were significantly increased in HF patients when compared with the control group. The correlation analysis indicated that circulating miRNA-21 correlated with the diagnosis and prognosis of HF. Therefore, it was suggested that circulating miRNA-21 has potential to become a novel biomarker of human HF.

\section{Acknowledgements}

The current study was supported by Youth Project (grant no. 2013ZRQN19) of the Natural Science Foundation of the First Affiliated Hospital of Xinjiang Medical University. The abstract has been previously published in the Proceedings of the 18th South China International Congress of Cardiology April 17-10, 2016.

\section{References}

1. Lee RC, Feinbaum RL and Ambros V: The C. elegans heterochronic gene lin-4 encodes small RNAs with antisense complementarity to lin-14. Cell 75: 843-854, 1993.

2. Wightman B, Ha I and Ruvkun G: Posttranscriptional regulation of the heterochronic gene lin-14 by lin- 4 mediates temporal pattern formation in C. elegans. Cell 75: 855-862, 1993.

3. Bartel DP: MicroRNAs: Genomics, biogenesis, mechanism and function. Cell 116: 281-297, 2004.

4. Gidlöf O, Smith JG, Miyazu K, Gilje P, Spencer A, Blomquist S and Erlinge D: Circulating cardio-enriched microRNAs are associated with long-term prognosis following myocardial infarction BMC Cardiovasc Disord 13: 12, 2013.

5. Marfella R, Di Filippo C, Potenza N, Sardu C, Rizzo MR, Siniscalchi M, Musacchio E, Barbieri M, Mauro C, Mosca N, et al: Circulating microRNA changes in heart failure patients treated with cardiac resynchronization therapy: Responders vs. Non-responders. Eur J Heart Fail 15: 1277-1288, 2013.

6. Ellis KL, Cameron VA, Troughton RW, Frampton CM, Ellmers LJ and Richards AM: Circulating microRNAs as candidate markers to distinguish heart failure in breathless patients. Eur J Heart Fail 15: 1138-1147, 2013.

7. Asangani IA, Rasheed SA, Nikolova DA, Leupold JH, Colburn NH, Post S and Allgayer H: MicroRNA-21 (miR-21) post-transcriptionally downregulates tumor suppressor Pdcd4 and stimulates invasion, intravasation and metastasis in colorectal cancer. Oncogene 27: 2128-2136, 2008.

8. Selcuklu SD, Donoghue MT and Spillane C: miR-21 as a key regulator of oncogenic processes. Biochem Soc Trans 37: 918-925, 2009.

9. Suárez Y, Fernández-Hernando C, Pober JS and Sessa WC: Dicer dependent microRNAs regulate gene expression and functions in human endothelial cells. Circ Res 100: 1164-1173, 2007.

10. Ji R, Cheng Y, Yue J, Yang J, Liu X, Chen H, Dean DB and Zhang C: MicroRNA expression signature and antisense-mediated depletion reveal an essential role of MicroRNA in vascular neointimal lesion formation. Circ Res 100: 1579-1588, 2007.
11. Roy S, Khanna S, Hussain SR, Biswas S, Azad A, Rink C, Gnyawali S, Shilo S, Nuovo GJ and Sen CK: MicroRNA expression in response to murine myocardial infarction: miR-21 regulates fibroblast metalloprotease-2 via phosphatase and tensin homologue. Cardiovasc Res 82: 21-29, 2009.

12. Zhang C: MicroRNomics: A newly emerging approach for disease biology. Physiol Genomics 33: 139-147, 2008.

13. Cheng Y and Zhang C: MicroRNA-21 in cardiovascular disease. J Cardiovasc Transl Res 3: 251-255, 2010.

14. Dong S, Cheng Y, Yang J, Li J, Liu X, Wang X, Wang D, Krall TJ, Delphin ES and Zhang C: MicroRNA expression signature and the role of microRNA-21 in the early phase of acute myocardial infarction. J Biol Chem 284: 29514-29525, 2009.

15. Thum T, Gross C, Fiedler J, Fischer T, Kissler S, Bussen M, Galuppo P, Just S, Rottbauer W, Frantz S, et al: MicroRNA-21 contributes to myocardial disease by stimulating MAP kinase signalling in fibroblasts. Nature 456: 980-984, 2008.

16. Shepler SA and Patel AN: Cardiac cell therapy: A treatment option for cardiomyopathy. Crit Care Nurs Q 30: 74-80, 2007.

17. Pruitt AL: Heart failure: It's not just for men. Crit Care Nurs Clin North Am 20: 327-341, 2008.

18. Chiong M, Wang ZV, Pedrozo Z, Cao DJ, Troncoso R, Ibacache M, Criollo A, Nemchenko A, Hill JA and Lavandero S: Cardiomyocyte death: Mechanisms and translational implications. Cell Death Dis 2: e244, 2011.

19. Thum T, Galuppo P, Wolf C, Fiedler J, Kneitz S, van Laake LW, Doevendans PA, Mummery CL, Borlak J, Haverich A, et al: MicroRNAs in the human heart: A clue to fetal gene reprogramming in heart failure. Circulation 116: 258-267, 2007.

20. Dong S, Ma W, Hao B, Hu F, Yan L, Yan X, Wang Y, Chen Z and Wang Z: microRNA-21 promotes cardiac fibrosis and development of heart failure with preserved left ventricular ejection fraction by up-regulating Bcl-2. Int J Clin Exp Pathol 7: 565-574, 2014.

21. Emerging Risk Factors Collaboration, Di Angelantonio E, Sarwar N, Perry P, Kaptoge S, Ray KK, Thompson A, Wood AM, Lewington S, Sattar N, et al: Major lipids, apolipoproteins, and risk of vascular disease. JAMA 302: 1993-2000, 2009.

22. van Holten TC, Waanders LF, de Groot PG, Vissers J, Hoefer IE, Pasterkamp G, Prins MW and Roest M: Circulating biomarkers for predicting cardiovascular disease risk; a systematic review and comprehensive overview of meta-analyses. PLoS One 8: e62080, 2013.

23. Rodseth RN, Lurati Buse GA, Bolliger D, Burkhart CS Cuthbertson BH, Gibson SC, Mahla E, Leibowitz DW and Biccard BM: The predictive ability of pre-operative B-type natriuretic peptide in vascular patients for major adverse cardiac events: An individual patient data meta-analysis. J Am Coll Cardiol 58: 522-529, 2011.

24. Liu L, Aguirre SA, Evering WE, Hirakawa BP, May JR, Palacio K, Wang J, Zhang Y and Stevens GJ: miR-208a as a biomarker of isoproterenol-induced cardiac injury in Sod2 $2^{+/-}$and C57BL/6J wild-type mice. Toxicol Pathol 42: 1117-1129, 2014.

25. Devaux Y, Vausort M, McCann GP,Zangrando J, Kelly D, Razvi N, Zhang L, Ng LL, Wagner DR and Squire IB: MicroRNA-150: A novel marker of left ventricular remodeling after acute myocardial infarction. Circ Cardiovasc Genet 6: 290-298, 2013.

26. Olivieri F, Antonicelli R, Lorenzi M, D'Alessandra Y, Lazzarini R, Santini G, Spazzafumo L, Lisa R, La Sala L, Galeazzi R, et al: Diagnostic potential of circulating miR-499-5p in elderly patients with acute non ST-elevation myocardial infarction. Int J Cardiol 167: 531-536, 2013

27. Zhang HL, Yang LF, Zhu Y, Yao XD, Zhang SL, Dai B, Zhu YP, Shen YJ, Shi GH and Ye DW: Serum miRNA-21: Elevated levels in patients with metastatic hormone-refractory prostate cancer and potential predictive factor for the efficacy of docetaxel-based chemotherapy. Prostate 71: 326-331, 2011.

28. Wang ZX, Bian HB, Wang JR, Cheng ZX, Wang KM and De W: Prognostic significance of serum miRNA-21 expression in human non-small cell lung cancer. J Surg Oncol 104: 847-851, 2011.

29. McMurray JJ, Adamopoulos S, Anker SD, Auricchio A, Böhm M, Dickstein K, Falk V, Filippatos G, Fonseca C, GomezSanchez MA, et al: ESC guidelines for the diagnosis and treatment of acute and chronic heart failure 2012: The Task Force for the Diagnosis and Treatment of Acute and Chronic Heart Failure 2012 of the European Society of Cardiology. Developed in collaboration with the Heart Failure Association (HFA) of the ESC. Eur J Heart Fail 14: 803-869, 2012. 
30. Adnan M, Morton G and Hadi S: Analysis of rpoS and bolA gene expression under various stress-induced environments in planktonic and biofilm phase using $2(-\Delta \Delta \mathrm{CT})$ method. Mol Cell Biochem 357: 275-282, 2011.

31. Truong QA, Januzzi JL, Szymonifka J, Thai WE, Wai B, Lavender Z, Sharma U, Sandoval RM, Grunau ZS, Basnet S, et al: Coronary sinus biomarker sampling compared to peripheral venous blood for predicting outcomes in patients with severe heart failure undergoing cardiac resynchronization therapy: The BIOCRT study. Heart Rhythm 11: 2167-2175, 2014.

32. Phelan D, Watson C, Martos R, Collier P, Patle A, Donnelly S, Ledwidge M, Baugh J and McDonald K: Modest elevation in BNP in asymptomatic hypertensive patients reflects sub-clinical cardiac remodeling, inflammation and extracellular matrix changes. PLoS One 7: e49259, 2012.

33. Luchner A, Stevens TL, Borgeson DD, Redfield M, Wei CM, Porter JG and Burnett JC Jr: Differential atrial and ventricular expression of myocardial BNP during evolution of heart failure. Am J Physiol 274: H1684-H1689, 1998.
34. Olivieri F, Spazzafumo L, Santini G, Lazzarini R, Albertini MC, Rippo MR, Galeazzi R, Abbatecola AM, Marcheselli F, Monti D, et al: Age-related differences in the expression of circulating microRNAs: miR-21 as a new circulating marker of inflammaging. Mech Ageing Dev 133: 675-685, 2012.

35. Olivieri F, Spazzafumo L, Bonafè M, Recchioni R, Prattichizzo F, Marcheselli F, Micolucci L, Mensà E, Giuliani A, Santini G, et al: MiR-21-5p and miR-126a-3p levels in plasma and circulating angiogenic cells: Relationship with type 2 diabetes complications. Oncotarget 6: 35372-35382, 2015.

36. Cardin S, Guasch E, Luo X, Naud P, Le Quang K, Shi Y, Tardif JC, Comtois P and Nattel S: Role for microRNA-21 in atrial profibrillatory fibrotic remodeling associated with experimental postinfarction heart failure. Circ Arrhythm Electrophysiol 5: 1027-1035, 2012.

37. Abraham WT, Fisher WG, Smith AL, Delurgio DB, Leon AR, Loh E, Kocovic DZ, Packer M, Clavell AL, Hayes DL, et al: Cardiac resynchronization in chronic heart failure. $\mathrm{N}$ Engl $\mathrm{J}$ Med 346: 1845-1853, 2002. 